\title{
De l'action collective des moteurs à l'ordre cellulaire
}

\section{François Nédélec Thomas Surrey}

F. Nédélec, T. Surrey : Laboratoire européen de biologie moléculaire. Cell Biology and Biophysics, Meyerhofstr. 1, 69117 Heidelberg, Allemagne.
L'organisation spatiale d'une cellule résulte des mouvements et des interactions des molécules qui la constituent. En organisant les filaments du cytosquelette dans la cellule, les protéines motrices jouent ici un rôle central. La synergie du réseau complexe de filaments interconnectés par les moteurs entraîne, par exemple, la séparation des chromosomes, ou le mouvement d'un macrophage. Une bonne connaissance du comportement individuel des moteurs est importante, mais la véritable difficulté est de comprendre leur action collective sur le réseau. Pour développer et tester les concepts permettant de déduire les propriétés globales d'un tel mélange de filaments et dè moteurs à partir des connaissances individuelles des molécules, il faut des outils qui en permettent l'étude quantitative et détaillée. Les reconstitutions in vitro à partir d'éléments purifiés et les simulations numériques ont cette aptitude.

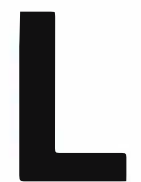

e mouvement, quelle que soit l'échelle à laquelle il se produit, est essentiel à la vie. Nous jouissons des forces produites par nos muscles, tissus faits de cellules contractiles très spécialisées. Certaines cellules peuvent aussi se déplacer individuellement, telles que les cellules du système immunitaire indispensables à notre survie (voir ce numéro pages 757 et 767), les spermatozoïdes, etc. De plus, qu'elles soient mobiles ou non, toutes les cellules eucaryotes sont le siège de nombreux mouvements internes qui sont à la base d'un ordre indispensable aux différentes fonctions du vivant. Par exemple, pour se diviser, la cellule construit une ossature appelée fuseau mitotique, destinée à rassembler les chromosomes, puis à les séparer en deux ensembles égaux qui formeront les génomes de deux cellules filles. Le cytosquelette inclut, par définition, I'ensemble des protéines qui participent à cet ordre cellulaire, et en particulier les fibres qui charpentent la cellule. Les structures qu'elles forment sont dynamiques. Ainsi, la séparation des chromosomes est la résultante d'une multitude de mouvements plus rapides qu'effectuent les constituants du fuseau mitotique. Peut-on comprendre l'action collective de ces protéines du cytosquelette, dont beau- coup sont maintenant connues et caractérisées, expliquer comment les nombreux mouvements qui se produisent à l'échelle moléculaire définissent l'ordre à l'échelle cellulaire ?

Avant d'étudier la synergie d'un ensemble de protéines, il faut d'abord nous familiariser avec les caractéristiques de leurs mouvements individuels. Les protéines ne mesurent que quelques nanomètres. Elles évoluent dans le cytoplasme de la cellule, dont la viscosité est environ 10 fois supérieure à celle de l'eau*, et ceci détermine les forces qui gouvernent leur mouvement. Le mouvement des objets macroscopiques qui nous sont familiers est soumis à l'inertie, et il est souvent relativement peu freiné par I'air. Par exemple, une balle de golf peut continuer son mouvement longtemps après qu'un joueur l'ait frappée, et parcourir ainsi une distance bien supérieure à sa taille. Au contraire, l'inertie des protéines est négligeable, car la friction du fluide qui les entoure les freine presque immédiatement**. Cependant, une protéine soluble ne reste jamais immobile. Elle est constamment heur-

\footnotetext{
* Pour simplifier, nous négligeons ici les comportements non newtoniens du cytosol de la cellule.

** En mécanique des fluides, cela s'appelle un régime à très petit nombre de Reynolds.
} 
Tableau I

VALEURS APPROCHÉES DES PARAMĖTRES INFLUENÇANT LE MOUVEMENT DES PARTICULES

\begin{tabular}{|c|c|c|c|c|}
\hline & $\begin{array}{l}\text { Protéine dans le } \\
\text { cytosol }\end{array}$ & $\begin{array}{c}\text { Vésicule dans le } \\
\text { cytosol }\end{array}$ & $\begin{array}{l}\text { Cellule eucaryote } \\
\text { dans l'eau }\end{array}$ & $\begin{array}{l}\text { Balle de golf } \\
\text { dans I'air* }\end{array}$ \\
\hline Taille $R(\mu \mathrm{m})$ & $2 \times 10^{-3}$ & $5 \times 10^{-2}$ & 10 & $2,1 \times 10^{+4}$ \\
\hline Masse M (mg) & $5 \times 10^{-17}$ & $5 \times 10^{-13}$ & $4 \times 10^{-6}$ & $4,6 \times 10^{+4}$ \\
\hline Mobilité $\mu$ (s/mg) & $2 \times 10^{+3}$ & $10^{+2}$ & 5 & $10^{-1}$ \\
\hline Coefficient de diffusion $D\left(\mu \mathrm{m}^{2} / \mathrm{s}\right)$ & 10 & $4 \times 10^{-1}$ & $2 \times 10^{-2}$ & $5 \times 10^{-4}$ \\
\hline Temps de freinage par friction (s & $10^{-13}$ & $5 \times 10^{-11}$ & $2 \times 10^{-5}$ & $\left(5 \times 10^{+3}\right)$ \\
\hline
\end{tabular}

La dispersion d'un ensemble de particules par diffusion en fonction du temps $t$ est $\left\langle x^{2}\right\rangle=2 D t$, où $D$ est le coefficient de diffusion. Celui-ci dépend de la mobilité des particules $\mu$ et de la température $T$ via $D=k_{B} T \mu$, avec $k_{B}$ la constante de Boltzman. La mobilité d'une sphère de rayon $R$ dans un fluide de viscosité $\eta$ est $1 /(6 \pi R)$. Pour une particule de vitesse $v$, la friction visqueuse est $F=v / \mu$. Si la particule est de masse $M$, le freinage par le fluide prend un temps M.n. Les valeurs du tableau sont approchées, et calculées pour une viscosité typique du cytosol, c'est-à-dire dix fois supérieure à celle de l'eau.

* La friction de l'air sur une balle de golf est plus compliquée que décrite ici. Le temps de freinage obtenu n'est pas réaliste, mais illustre bien la dif-

tée par les molécules du fluide en perpétuel mouvement, et ces collisions la poussent au hasard dans une direction ou une autre. Le mouvement qui en résulte est une "marche" aléatoire, décrite par les formules mathématiques qui régissent les lois de la diffusion (voir Tableau ). La diffusion est indispensable, car elle permet aux protéines d'explorer efficacement l'espace. Par ailleurs, la diffusion se produit avec une chance égale dans toutes les directions, et il en résulte une dispersion des protéines qui doit être surmontée pour qu'un ordre puisse apparaître. C'est le rôle de certaines protéines.

Les cellules eucaryotes possèdent des enzymes mécaniques appelées protéines motrices. Elles sont capables de se déplacer le long des fibres du cytosquelette, par pas successifs, en consommant l'énergie de molécules $d^{\prime}$ ATP [1]. Elles peuvent produire des forces de quelques pico-Newton $\left(10^{-12} \mathrm{~N}\right)[2]$, et cela est largement suffisant pour tirer des charges bien plus importantes que le moteur lui même, par exemple des vésicules (voir ce numéro, p. 745) ou même d'autres filaments du cytosquelette. Reste à savoir si le moteur peut surmonter le processus de diffusion évoqué plus haut pour réaliser ce mouvement actif. Pour cela, nous devons comparer la distance parcourue lors du mouvement actif du moteur avec celle qu'occasionne sa dispersion due à la diffusion. Le résultat dépend (1) des efficacités intrinsèques de ces deux mouvements, définies respecti- vement par la vitesse du moteur, et par son coefficient de diffusion; (2) du temps que le moteur passe dans ces deux états: accroché, état associé au mouvement* et détaché, état associé à une diffusion libre en solution. Illustrons cela par un exemple simple: la vitesse d'un moteur est de l'ordre de $1 \mu \mathrm{m} / \mathrm{s}$, et son coefficient de diffusion dans le cytosol est de l'ordre de $10 \mu \mathrm{m}^{2} / \mathrm{s}$ (voir Tableau 1). En principe, le moteur peut parcourir une distance de $20 \mu \mathrm{m}$, c'est-à-dire traverser une cellule, en 20 secondes. En fait, un moteur ne parcourt jamais une telle distance d'une seule traite, mais seulement après plusieurs phases de détachement, puis attachement, et lorsqu'il n'est pas attaché, le moteur diffuse. Si le temps pendant lequel le moteur est attaché était égal au temps pendant lequel il se détache, la diffusion serait alors de $20 \mu \mathrm{m}$, et occulterait partiellement le transport. En revanche, si le moteur transporte une vésicule (figure 1A), comme c'est souvent le cas dans la réalité, sa diffusion pendant ce laps de temps ne serait que de $4 \mu \mathrm{m}$, et ce serait le transport qui serait alors le plus efficace, au bénéfice de la cellule. Cependant, sur une plus courte distance, la diffusion est à nouveau importante: pour déplacer une vésicule de $1 \mu \mathrm{m}$, notre moteur nécessite 1 seconde, et la diffusion est alors de

* La diffusion des filaments, qui contribue alors au mouvement du moteur qui lui est accroché, est négligeable.
$1 \mu \mathrm{m}$. Ceci s'explique parce que mouvement dirigé et diffusion sont de nature très différente, et que leur efficacité comparée dépend également de l'échelle considérée. La diffusion est plus efficace à petite échelle, et le transport plus efficace à grande échelle. Pour rester simple, nous avons supposé l'égalité des temps pendant lequel le moteur est attaché et détaché. En toute rigueur, cela est défini par une constante d'équilibre et par la concentration locale de filaments. Pour cette raison, I'analyse complète est plus compliquée qu'elle n'est décrite ici. Pour passer outre à ce problème de diffusion, qui s'oppose à leur mouvement, les moteurs semblent adopter deux stratégies: certains passent la majeure partie du temps dans un état accroché, effectuant de nombreux pas avant de se détacher - ces moteurs sont dits "tenaces" ou processive en anglais. Les autres moteurs, qui ne restent accrochés qu'un court instant, compensent cet inconvénient en agissant en grand nombre. Leur stratégie est alors de s'associer en groupe, c'est le cas de la myosine qui forme des filaments de moteurs [3]. Les propriétés physiques des moteurs leur permettent donc d'être efficaces même dans un environnement dominé par la friction fluide du solvant et la diffusion des molécules.

Voyons maintenant comment le cytosquelette de la cellule est organisé, et en particulier comment les microtubules et les filaments d'actine $[4,5]$ sont disposés dans la cellule. La 

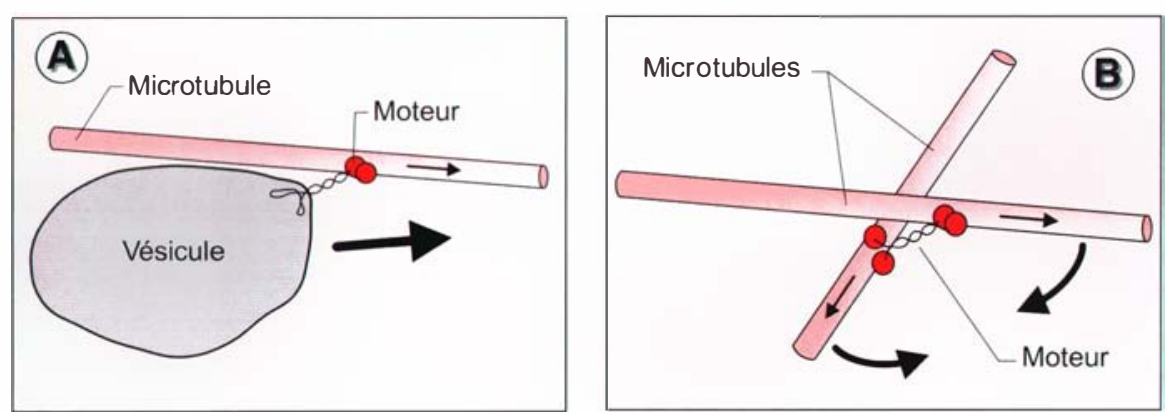

Figure 1. Exemple de fonction des moteurs dans la cellule. A. Un moteur transporte une vésicule le long d'un microtubule. B. Un moteur oligomérique peut "organiser" les filaments, en les déplaçant l'un par rapport à l'autre.

disposition de ces fibres, sur lesquelles se déplacent les moteurs, n'est pas aléatoire: elle est souvent spécifique d'un type de cellule et de son état, notamment lors de la mitose (figure $2 A, 2 B$ ). Comment se forment ces structures? II s'avère que les protéines motrices ont ici un rôle crucial [6], car elles sont aussi capables de transporter des fibres du cytosquelette le long d'autres fibres (figure 1B). L'action des moteurs contribue alors à l'organisation de la cellule. Ainsi, les filaments déterminent le mouvement des moteurs, et les moteurs peuvent déplacer les filaments. Ils s'organisent donc mutuellement, et cela confère au cytosquelette une dynamique remarquable. II est important ici de réaliser que l'ordre cellulaire - à l'échelle du micromètre - est la conséquence $d$ 'un très grand nombre d'interactions élémentaires entre les constituants du cytosquelette - à l'échelle du nanomètre -, interactions qui ne sont pas a priori coordonnées. L'organisation du cytosquelette est issue de l'action cumulée des multiples mouvements de ses protéines, accroissant leur potentiel, et cachant une partie de la stochasticité des interactions. Illustrons ceci par un exemple concret.

La formation du fuseau mitotique est un exemple classique de morphogenèse [7]. II est formé de chromosomes, de microtubules et des protéines associées. Dans une cellule eucaryote qui s'apprête à se diviser, la phosphorylation de nombreuses protéines affecte les propriétés des microtubules et l'activité de nombreux moteurs [8]. En quelques minutes, la disposition radiale de longs microtubules typique d'une cel-
Iule au repos est remplacée par un fuseau bipolaire de microtubules très dynamiques (figure 2). Ce fuseau permet à la cellule de partager ses chromosomes équitablement, leurs mouvements étant engendrés par la dynamique des filaments et par les moteurs. Attention! Les moteurs ne font pas que tirer les chromosomes en s'appuyant sur le réseau de microtubules, ils sont aussi à la base de ce réseau. Après la division, I'agencement radial des microtubules est rétabli dans les deux cellules filles, et le cycle peut recommencer. Les techniques dont nous disposons pour étudier la cellule sont multiples. La microscopie optique permet d'observer la cellule vivante en temps réel et la fluorescence de connaître la localisation des protéines marquées. L'effet de la création de mutations de protéines, de la suppression ou de la surexpression de ces protéines peut aussi être observé. En utilisant des extraits cellulaires, on peut même reconstituer in vitro la formation du fuseau, ce qui offre l'avantage d'un système ouvert facilement manipulable biochimiquement. Ces expériences peuvent suggérer la fonction d'une protéine dans le contexte extrêmement complexe du cytosol, et ont ainsi clairement démontré l'importance de certains moteurs. En revanche, la composition des échantillons reste inconnue, et il est impossible de savoir si les protéines identifiées, qu'on sait être nécessaires, sont aussi suffisantes. De plus, la complexité de ces systèmes rend leur compréhension laborieuse.

Afin d'analyser la "fonction organisatrice" des moteurs, il est souhaitable d'étudier l'évolution d'un ensemble de moteurs et de filaments dans un système de complexité minimale. La construction "artificielle" d'une partie de la complexité cellulaire à partir de quelques protéines purifiées [10] est une approche complémentaire à I'étude de l'ensemble de la cellule, avec toute sa complexité et sa composition encore partiellement inconnue. Les mélanges de moteurs et de microtubules purifiés permettent ainsi de créer des systèmes modèles accessibles à l'analyse. Dans ces expériences, les moteurs, par exemple des kinésines de la famille BimC essentielles au fuseau mitotique, sont solubles et oligomériques. Les moteurs oligomériques peuvent organiser les microtubules en s'accrochant simultanément sur deux d'entre eux, et en les déplaçant l'un par rapport à l'autre (figure 1B). En d'autres termes, ils forment des liens mobiles et actifs entre les filaments. Nous pouvons bien imaginer l'action d'un lien sur une paire isolée de microtubules, mais quel est le résultat d'une multitude de liens sur un réseau entier de filaments ?

Les expériences montrent que les moteurs oligomériques organisent les microtubules en différentes structures: asters, vortex ou faisceaux (figures 2C, 2D). II est déjà remarquable que ce simple mélange de microtubules et de moteurs ait gardé la capacité - essentielle - du cytosquelette à former différentes structures. Bien que les arrangements de microtubules, une fois formés dans les expériences, semblent statiques, ces structures sont néanmoins intrinsèquement dynamiques. Seules les microtubules sont stationnaires, les moteurs, eux, y sont en perpétuel mouvement. Ainsi, dans le vortex, ils tournent en continu autour de l'anneau central de microtubules. Les structures formées dans l'expérience sont des états stationnaires dans lesquels les composants sont animés et consomment de l'énergie. Ce sont donc les interactions dynamiques des protéines qui décident quelle structure se forme; à leur tour, ces interactions sont déterminées par les propriétés des protéines et leur concentration. Dans notre expérience, toutes les constantes cinétiques qui caractérisent les interactions et, bien sûr, la concentration des protéines sont connues. Cepen- 

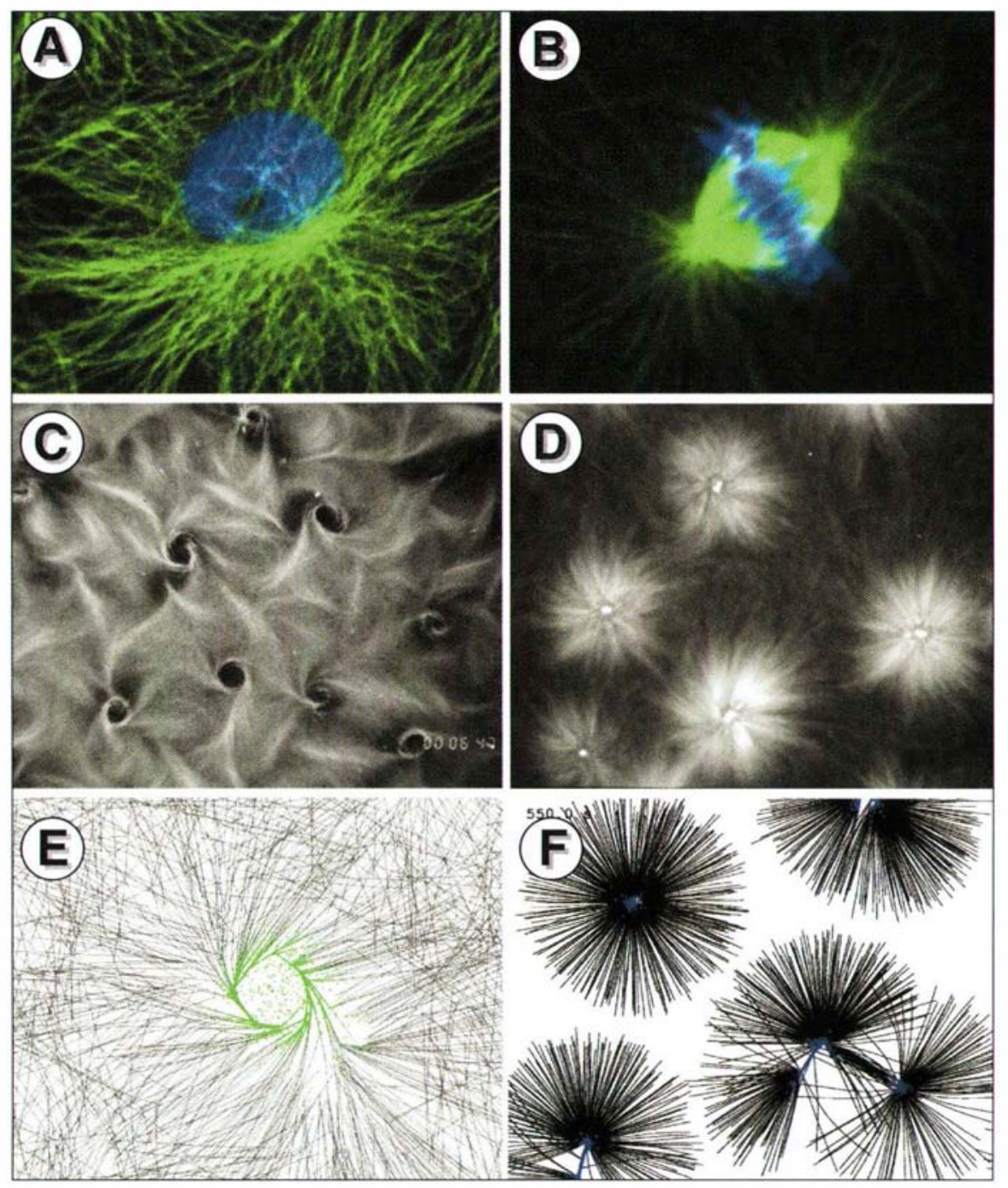

Figure 2. Exemples d'arrangements de microtubules dans la cellule, dans des mélanges purifiés in vitro, et dans les simulations numériques. Cellule eucaryote en interphase (A) et en mitose (B) vue en microscopie de fluorescence; les microtubules sont marquées en vert, I'ADN en bleu (I. Vernos et $T$. Wittmann). Vortex de microtubules (C) et asters (D) produits par auto-organisation d'un mélange de microtubules et de moteurs, observé en microscopie de champ noir. Les microtubules apparaissent en blanc, les moteurs sont invisibles. Vortex de microtubules (E) et asters en formation (F) obtenu par simulation numérique, les microtubules sont en noir, les moteurs sont représentés par des points colorés.

dant, elles ne permettent pas de connaître immédiatement I'organisation des microtubules qui en résulte. Pour comprendre pourquoi certains motifs se forment et pas d'autres, il nous faut en quelque sorte extraire l'information concernant cette organisation qui est cachée dans ces chiffres.

La simulation numérique accomplit cette tâche. Elle repose sur une modélisation quantitative du comportement stochastique individuel des protéines et de leurs interactions. En utilisant les valeurs connues des constantes cinétiques des moteurs et des microtubules - coefficients de diffusion, vitesse de déplacement, probabilités d'attachement et de détachement, etc. -, la simulation permet tout d'abord de reproduire les structures observées dans l'expérience (figures 2E, 2F). Puisque chacun de ces paramètres peut être choisi à loisir, la simulation permet surtout d'étudier séparément l'influence de chacune des caractéristiques des protéines. Cela permet, par exemple, de démontrer que la capacité des moteurs oligomériques à former des asters est très sensible à leur comportement à l'extrémité des filaments. Si les moteurs restent accrochés après avoir atteint le bout des microtubules, cela favorise la formation d'asters. En revanche, si les moteurs se détachent à l'extrémité des microtubules, des vortex seront créés. La simulation permet donc d'étudier en détail l'effet des propriétés des individus et de leurs interactions sur l'ordre et les propriétés de l'ensemble. De plus, les simulations reproduisent tous les événements qui conduisent à l'évolution du système. Elles permettent par exemple de quantifier en détail le mouvement des moteurs ou la consommation d'énergie des structures dynamiques, choses difficilement accessibles à l'expérimentateur. Cela multiplie le pouvoir d'investigation du chercheur et facilite la compréhension de ces systèmes. Enfin, une fois confirmée, la simulation acquiert un pouvoir prédictif qui s'ajoute alors à notre intuition.

L'auto-organisation des moteurs et des microtubules en régime stationnaire est fondamentalement différente de l'auto-assemblage de protéines. Par exemple, on parle d'auto-assemblage de la capside de nombreux virus: les pièces de la capside se rencontrent par diffusion, et s'associent durablement ou non suivant leur affinité [10]. Celle-ci est définie par la forme géométrique des constituants, ainsi que par leurs motifs d'attraction ou de répulsion. L'assemblage final est statique, il correspond à un minimum de l'énergie d'interaction globale. Au contraire, I'auto-organisation repose sur une consommation continue d'énergie, même lorsque l'état est stationnaire. Les structures formées ne réalisent pas d'équilibre thermodynamique et sont largement déterminées par les constantes cinétiques des interactions moléculaires. $C^{\prime}$ est le cas de nombreux autres systèmes plus classiques: les tornades dans l'atmosphère, ou les motifs en spirales produits par réaction/diffusion $[11,12]$ de composés chimiques. Ces structures hors d'équilibre sont déterminées principalement par la dynamique collective des molécules présentes, et non par leurs 
réactions d'attraction/répulsion statiques. Une qualité générique de ces systèmes est leur capacité à produire des formes sur une échelle bien plus grande que la taille de leurs constituants. C'est exactement ce qui est observé dans le cas du cytosquelette, ou des systèmes biologiques en général. L'exemple des microtubules et des moteurs montre clairement qu'en plus de l'auto-assemblage - qui conduit à la formation des microtubules -, I'auto-organisation participe de manière cruciale à l'établissement de l'ordre cellulaire. Cela nous conduit à décrire le cytosquelette comme un système dynamique hors d'équilibre capable de résider dans certains état stationnaires, ou de transiter entre ces états.

Nous pouvons maintenant considérer le mouvement d'une cellule entière, car les deux propriétés caractéristiques d'un état stationnaire hors d'équilibre, d'une part un ordre conservé, et d'autre part une dynamique sous-jacente, sont exactement celles qui sont nécessaires au mouvement cellulaire. Le mouvement rapide des cellules n'est pas gouverné par les microtubules, mais par les filaments d'actine. Lorsque, par exemple, un macrophage se déplace, sa forme reste relativement constante, mais les filaments d'actine, qui produisent la force motrice, sont très dynamiques et renouvelés rapidement. L'actine polymérise vers I'avant, sur le front de la cellule, puis ces filaments se contractent sous l'action des moteurs faits de myosines, et enfin dépolymérisent plus en arrière de la cellule [13]. Un autre exemple de ce procédé est celui des fragments de kératocyte de poisson obtenus par traitement chimique |14] (figure 3). Des filaments du cytosquelette, ils ne contiennent que l'actine: ils ont perdu microtubules (et noyau) en se détachant du reste de la cellule. Malgré tout, ils sont toujours capables de se déplacer! Plus remarquable encore, on peut "animer» un fragment immobile en le poussant brièvement sur un côté (figure 3). La force appliquée est insuffisante pour déplacer le fragment de cellule, mais elle déforme son contour rond en une forme asymétrique, et ce changement entraîne le déplacement du fragment cellulaire qui, en quelque sorte, se déplace de lui-même. Le réseau d'actine semble glisser d'un état stationnaire vers un autre: le "turnover » symétrique de l'actine dans le fragment à l'arrêt devient asymétrique et propulse l'ensemble. Le changement de forme du fragment force les filaments à se réorganiser, ce qui crée une nouvelle dynamique de l'actine. Cet exemple, pour lequel le comportement est également déterminé par

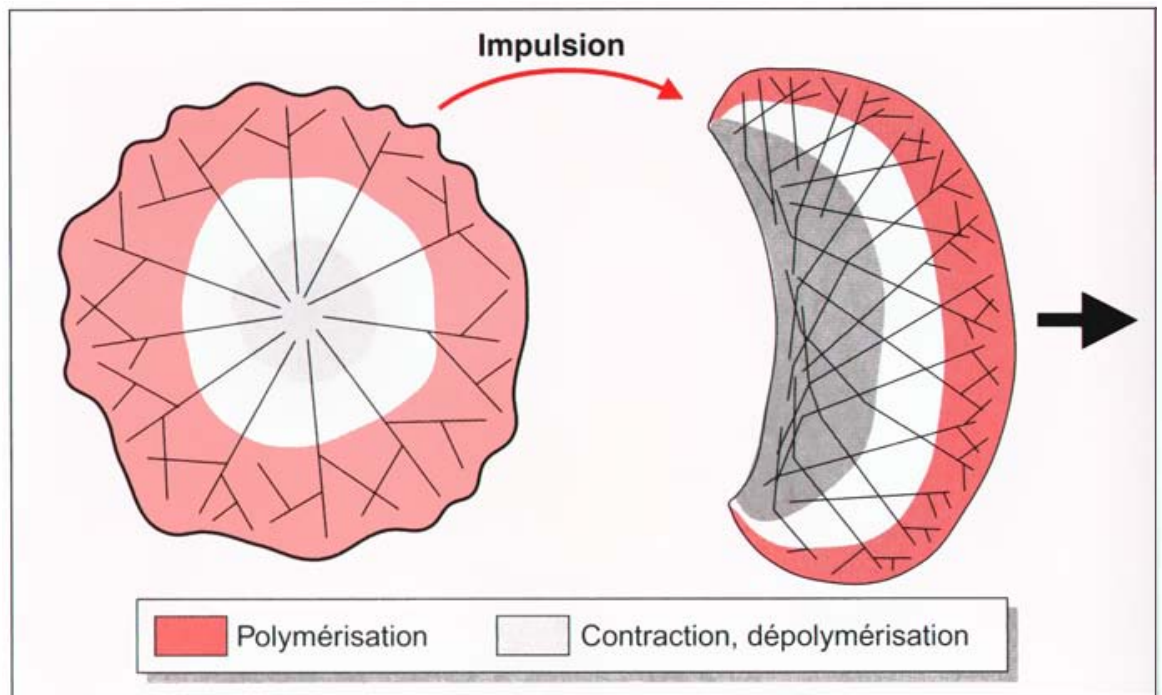

Figure 3. Schéma d'un fragment de kératocyte d'après [16]. II est représenté dans son état immobile (gauche) ou mobile (droite), avec respectivement un turn-over symétrique ou asymétrique des filaments d'actine (lignes). Le mouvement $d^{\prime} u n$ fragment immobile peut être induit par application d'une force brève, ici sur la gauche du fragment. la dynamique des filaments et l'action de moteurs, montre que l'ordre du système affecte directement la dynamique de ses protéines. Avec l'autoorganisation des microtubules et des moteurs, nous avons vu l'inverse: la dynamique des constituants détermine l'ordre global. Ensemble, ces deux exemples montrent que la dynamique des constituants et I'organisation du système sont indissociables et ne peuvent pas êtres compris séparément. Dynamique et ordre ne sont que deux aspects d'un seul et même système.

\section{Conclusions}

Dans cet article, nous nous sommes intéressés au rôle des moteurs dans l'organisation du réseau de filaments du cytosquelette. II est important de rappeler l'aspect collectif de cette action: de la synergie des interactions dépend le choix de la structure finale. Dans l'exemple d'un mélange de microtubules et de moteurs, nous avons démontré comment cette dynamique peut être reproduite, soit par des mélanges de protéines purifiées, soit par simulation numérique. Ces méthodes, aux avantages complémentaires, sont indispensables pour aborder les systèmes complexes, comme ceux présents dans la cellule. Nous avons insisté sur le caractère intrinsèquement dynamique des arrangements stationnaires obtenus par auto-organisation, et conclu qu'en fait, dynamique et ordre sont intimement liés dans ces structures hors d'équilibre. Considérer le caractère dynamique et stationnaire des états du cytosquelette devrait nous aider à en expliquer le fonctionnement. Pour ceci, il ne suffira plus d'identifier et de caractériser la fonction de protéines isolées, il faudra aussi développer les outils et les concepts qui permettront d'en comprendre l'action collective

\section{RÉFÉRENCES}

1. Howard J. The movement of kinesin along microtubules. Annu Rev Physiol 1996 ; 58 : 703-29.

2. Svoboda K, Block SM. Force and velocity measured for single kinesin molecules. Cell $1994 ; 77: 773-84$. 


\section{RÉFÉRENCES}

3. Hackney DD. The kinetic cycles of myosin, kinesin, and dynein. Annu Rev Physiol $1996 ; 58: 731-50$.

4. Desai A, Mitchison TJ. Microtubule polymerization dynamics. Annu Rev Cell Dev Biol 1997; 13: 83-117.

5. Higgs HN, Pollard TD. Regulation of actin polymerization by Arp $2 / 3$ complex and WASp/Scar proteins. I Biol Chem 1999; $274: 32531-4$.

6. Walczak CE, Vernos 1., Mitchison TJ, Karsenti $E$, Heald R. A model for the proposed roles of different microtubule-based motor proteins in establishing spindle bipolarity. Curr Biol 1998; 8: 903-13.

7. Heald R, Tournebize R, Blank $T$, et al. Self-organization of microtubules into bipolar spindles around artificial chromosomes in Xenopus egg extracts. Nature 1996;382 $420-5$.

8. Cassimeris L. Accessory protein regulation of microtubule dynamics throughout the cell cycle. Curr Opin Cell Biol P999. 11 : 134-41.

9. Nédélec FJ, Surrey T, Maggs AC, Leibler $S$. Self-organization of microtubules and motors. Nature 1997; 18:305-8.
10 . Inoue $\mathrm{S}$. The role of self-assembly in the generation of biological form. In developmental order: its origin and regulation. 40th Symposium of the Society for Developmental Biologists, S. Subtelny, P.B. Green, eds. New York: Alan R. Liss, Inc, 1982 : 35-30.

11. Turing AM. The chemical basis of morphogenesis. Phil Trans Roy SoC (London) 1952 ; B237 : 37-72.

12. Prigogine I, Nicolis G. On symetry-breaking instabilities in dissipative systems. J Chem Phys 1967; 46: 3542-50.

13. Cramer LP, Mitchison TJ. Actin-based cell motility and cell locomotion. Cell 1996: $84: 371-9$.

14. Verkhovsky AB, Svitkina TM, Borisy GG. Self-polarization and directional motility of cytoplasm. Curr Biol 1999; 9: 11-20.

TIRÉS À PART

F. Nédélec.

\section{ms 2000}

\section{Summary}

How individual random molecular motions create an ordered detection

Molecular motions are essential for the generation of order inside cells. Motor proteins play here a crucial role by organizing the filaments of the cytoskeleton in space. The dynamic of the network of filaments interconnected by motors form the basis of processes like chromosomes segregation and cell motility. A good knowledge of how individual motors behave in their world of small scales is important. However, the real difficulty is to understand their collective action on the entire filament network. To develop and test concepts which address the properties of filament and motor mixtures based on the characteristics of their components, we need tools that allow their quantitative study. In vitro reconstitution experiments and numerical computer simulations have the potential to achieve this goal.

\section{Université Paris VI - Hôtel - Dieu - Institut Benjamin-Délessert}

\section{JOURNÉE ANNUELLE DE NUTRITION ET DE DIÉTÉTIQUE}

\begin{tabular}{ll}
\hline Fondateurs : & Henri Bour, Maurice Dérot, Guy Héraud \\
\hline Président : & Professeur Bernard Guy-Grand \\
Vice-Présidents : & Professeur Arnaud Basdevant \\
& Professeur Bernard Messing \\
& Professeur Gérard Slama \\
Secrétaire Générale : & Marie-France Carrié-Moyal \\
Membre d'Honneur : & Docteur Michel Rathery \\
\hline
\end{tabular}

$41^{\mathrm{e}}$ Journée Annuelle de Nutrition et de Diététique

Vendredi 26 janvier 2001

CNIT-PARIS/LA DÉFENSE

Matinée : "De l'obésité de l'enfant à l'obésité de l'adulte » Après-midi : «Allergie alimentaire : reconnaître, prévenir, traiter ».

Organisation-Secrétariat :

Claire Bosc - 30, rue de Lübeck 75116 Paris - Tél. : 33145534169 Fax : 33144051337 